\title{
Autoavaliação de sintomas vocais e desconforto no trato vocal em indivíduos com diabetes mellitus tipo 1
}

\author{
Self-assessment of vocal symptoms and vocal tract discomfort in \\ individuals with type 1 diabetes mellitus
}

\author{
Laiana Weschenfelder ${ }^{1}$ (1), Vanessa Veis Ribeiro² (1), Ana Paula Dassie Leite ${ }^{1}$ (1)
}

\section{RESUMO}

Objetivo: Analisar a autoavaliação de sintomas vocais e do desconforto no trato vocal em indivíduos com e sem diabetes mellitus tipo 1 e analisar a influência do tempo de diagnóstico da doença e da forma de administração de insulinoterapia na autoavaliação. Métodos: Participaram 60 indivíduos, divididos em dois grupos: Grupo I (G1) - 30 indivíduos com diagnóstico de diabetes mellitus tipo 1; Grupo II (G2) - 30 indivíduos sem a doença. Para avaliação dos desfechos, os participantes responderam às questões da Escala de Sintomas Vocais e da Escala de Desconforto no Trato Vocal. Os participantes do G1 responderam também a um questionário sobre o diagnóstico e do tratamento médico da diabetes mellitus tipo 1 . Os dados foram analisados por meio de estatística descritiva e inferencial. Resultados: não houve diferença entre os grupos para a autoavaliação de sintomas vocais e desconforto no trato vocal. Em indivíduos do G1, foi encontrada correlação positiva moderada entre o tempo de diagnóstico e as variáveis frequência de queimação, intensidade de queimação e frequência de garganta sensível. Além disso, houve valores significativamente maiores de frequência e intensidade de garganta sensível e irritada em indivíduos que referiram realizar insulinoterapia utilizando bomba de infusão, em relação aos que mencionaram aplicações ao longo do dia. Conclusão: indivíduos com diabetes mellitus tipo 1 apresentam baixa sintomatologia vocal e desconforto no trato vocal. Porém, as características da doença referentes ao tempo de diagnóstico e a forma de administração de insulinoterapia influenciam a percepção da frequência e da intensidade de desconforto no trato vocal.

Palavras-chave: Autoavaliação; Diabetes mellitus; Distúrbios da voz; Laringe; Voz

\begin{abstract}
Purpose: To analyze the self-assessment of voice symptoms and vocal tract discomfort in individuals with and without Type 1 Diabetes Mellitus (T1D) and to analyze the influence of the time of diagnosis and the type of insulin therapy in the self-assessment. Methods: Sixty individuals participated, divided into two groups: Group I (G1) - 30 individuals with T1D; Group II (G2) - 30 individuals without T1D. The participants responded to the Voice Symptom Scale (VoiSS) and Vocal Tract Discomfort Scale (VTD). G1 participants also answered to a questionnaire to obtain data of the diagnosis and medical treatment of T1D. The data were analyzed using descriptive and inferential statistics. Results: There was no difference between groups for self-assessment of voice symptoms and vocal tract discomfort. In G1, a moderate positive correlation was found between time of diagnosis and the variables: burning frequency, burning intensity and frequency of soreness. In addition, there were significantly higher values of frequency and intensity of soreness and irritated throat in individuals who reported performing insulin therapy using an infusion pumps compared to those who realize multiple daily injections. Conclusion: Individuals with T1D have few vocal symptoms and vocal tract discomfort; they are not different from the control group. However, characteristics of the disease such as time of diagnosis and type of insulin therapy have influence in the perception of the frequency and intensity of some vocal tract discomfort.
\end{abstract}

Keywords: Self-assessment; Diabetes mellitus; Voice disorders; Larynx; Voice

\footnotetext{
Trabalho realizado na Universidade Estadual do Centro-Oeste - UNICENTRO - Irati (PR), Brasil.

${ }^{1}$ Universidade Estadual do Centro-Oeste - UNICENTRO - Irati (PR), Brasil.

${ }^{2}$ Universidade Federal de Sergipe - UFS - Lagarto (SE), Brasil.

Conflito de interesses: Não.

Contribuição dos autores: LW delimitação do estudo, coleta e análise de dados, redação do estudo; VVR análise de dados, redação e revisão do estudo; APDL delimitação do estudo, análise de dados, redação e revisão do estudo.

Financiamento: Nada a declarar.

Autor correspondente: Ana Paula Dassie Leite. E-mail: pauladassie@hotmail.com

Recebido: Abril 09, 2020; Aceito: Maio 19, 2020
} 


\section{INTRODUÇÃO}

A diabetes mellitus (DM) é uma doença sistêmica crônica, decorrente do excesso de glicose na corrente sanguínea e da produção deficiente de um hormônio protético (insulina) pelo pâncreas, que é responsável por manter os níveis normais de glicose no sangue ${ }^{(1)}$. A DM pode ser segmentada em tipo 1 (DM1) e tipo 2 (DM2), de acordo com o motivo da falta de insulina no organismo. A DM1 é causada por uma reação autoimune, que resulta na produção ineficiente de insulina pelo pâncreas, enquanto na DM2, o pâncreas produz insulina, mas o organismo apresenta resistência à metabolização da insulina.

A DM1 ou DM insulinodependente é caracterizada pela falta da insulina ${ }^{(2)}$. É considerada a segunda doença crônica mais frequente em crianças e adolescentes brasileiros ${ }^{(1,3)}$. Na DM1, é realizada a administração de insulina de forma injetável, subcutânea, a fim de manter normais as concentrações séricas de insulina ${ }^{(4)}$. Desta forma, o tratamento da DM1 é realizado por meio da aplicação de insulina e o controle do nível de glicose no sangue. Os tipos de administração da insulinoterapia mais comuns ocorrem por meio do uso de bomba de infusão ou aplicações ao longo do dia e a escolha do tipo e esquema de tratamento dependem de características da doença e do portador ${ }^{(5)}$.

Alguns sintomas gerais da DM1 são fadiga, cansaço, sede e fome intensos ${ }^{(2)}$. Além disso, há relatos, na literatura, de associação da DM com refluxo laringofaríngeo (RLF) ${ }^{(6)} \mathrm{e}$ distúrbio sensorial laríngeo ${ }^{(6,7)}$. Estudo que relacionou DM2 e sintomas laringofaríngeos mostrou maior frequência de pigarro, sensação de bolo na garganta e tosse nesses pacientes, em comparação aos não doentes (grupo controle) ${ }^{(6)}$.

Acredita-se que é necessária a obtenção de evidências sobre a possível influência de variáveis relacionadas à DM1 nos sintomas e desconfortos vocais, a fim de se contribuir para a compreensão sobre as queixas desses pacientes quando buscam por avaliação e tratamento na área de voz. Além disso, considera-se importante relacionar os resultados da autoavaliação com dados da doença que poderiam ser responsáveis por tais manifestações.

Assim, o presente estudo apresentou como objetivos: analisar a autoavaliação de sintomas vocais e do desconforto no trato vocal em indivíduos com e sem DM1 e analisar a influência do tempo de diagnóstico da doença e da forma de administração de insulinoterapia na autoavaliação.

\section{MÉTODOS}

Trata-se de estudo observacional, transversal e analítico, aprovado pelo Comitê de Ética em Pesquisa da UNICENTRO (n.3.231.344). Os participantes foram recrutados por meio de divulgação em mídias sociais. A seleção amostral e a coleta de dados foram realizadas on-line, por meio de um link disponibilizado no momento do recrutamento, que direciona para o preenchimento da pesquisa, via Google Forms ${ }^{\circledR}$.

Adotaram-se os seguintes critérios gerais de inclusão: ambos os sexos, idades entre 18 e 54 anos e assinatura do Termo de Consentimento Livre e Esclarecido, concordando em participar da pesquisa. Além disso, considerou-se como critério de inclusão específico dos grupos a autorreferência de diagnóstico de DM1. Como critérios gerais de exclusão, foram considerados tabagismo, relato de diagnóstico prévio de alteração laríngea e/ou distúrbio vocal, problemas respiratórios, alterações neurológicas ou metabólicas/hormonais, queixa auditiva e utilização profissional da voz. Para selecionar os participantes, foi aplicado um questionário com perguntas diretas sobre os critérios de seleção. Alguns dados coletados nesse questionário foram utilizados para caracterizar a amostra incluída.

Dessa forma, a amostra de conveniência do presente estudo foi composta por 60 indivíduos, divididos em dois grupos: Grupo I (G1) - 30 indivíduos com DM1, média de idade de 31 anos e 7 meses, 16 do sexo masculino e 14 do sexo feminino; Grupo II (G2) - 30 indivíduos que não possuíam a doença, média de idade de 32 anos, 16 do sexo masculino e 14 do sexo feminino. Não houve diferença entre os grupos quanto à idade $(\mathrm{p}=0,497$ - Teste-T Independente) e sexo $(\mathrm{p}=1,000$ - Teste Exato de Fisher).

Foram avaliados dois desfechos: autoavaliação de sintomas vocais e autoavaliação de desconforto no trato vocal.

A Escala de Sintomas Vocais (ESV) ${ }^{(8)}$ é um instrumento validado no português brasileiro para mensurar a percepção de sintomas vocais e seu impacto no dia a dia. O instrumento é composto por 30 itens, pontuados quanto à frequência em uma escala de Likert de 0 (zero) (nunca) a 4 (sempre). Os itens analisam os fatores limitação, físico e emocional, além de um escore total. O cálculo do instrumento é feito por somatória simples das questões de cada fator.

A Escala de Desconforto no Trato Vocal (EDTV) ${ }^{(9)}$ é um instrumento traduzido para o português brasileiro ${ }^{(10)}$ para mensurar a frequência e intensidade do desconforto no trato vocal. $\mathrm{O}$ instrumento é composto por 16 itens, referentes à intensidade e frequência de oito desconfortos no trato vocal, pontuados em uma escala de Likert de 0 (zero) (nunca/nenhuma) a 6 (sempre/extrema). A análise das questões é individual e por somatória simples da frequência e da intensidade dos desconfortos.

Os participantes do G1 responderam, também, a um questionário elaborado pelos autores deste estudo para obter-se dados sobre o tempo de diagnóstico (em anos) e a forma de administração de insulinoterapia.

Os dados foram analisados estatisticamente, de forma descritiva e inferencial. $\mathrm{Na}$ análise descritiva, foram calculadas medidas de tendência central, variabilidade e posição. Para realizar a análise inferencial, os dados quantitativos foram submetidos à análise de normalidade com o Teste Shapiro-Wilk. A análise inferencial de comparação da variável normal entre dois grupos independentes foi realizada com o Teste-T Independente e a de comparação das variáveis quantitativas não normais, com o Teste de Mann-Whitney. A correlação entre variáveis quantitativas não normais foi realizada com o Teste de Correlação de Spearman. Foi analisada a força de correlação ${ }^{(11)}$. A associação entre duas variáveis qualitativas nominais de duas categorias foi realizada com o Teste Exato de Fisher. Foi utilizado nível de significância de 5\%.

\section{RESULTADOS}

Não houve diferença entre os grupos para a autoavaliação de sintomas vocais e desconforto no trato vocal (Tabela 1 ).

Considerando-se apenas o G1, o tempo mediano de diagnóstico da DM1 foi de 15 anos e foram mais frequentes os participantes 
Tabela 1. Comparação da autoavaliação de sintomas vocais e do desconforto no trato vocal entre indivíduos com e sem diabetes mellitus tipo 1

\begin{tabular}{|c|c|c|c|c|c|c|c|c|c|}
\hline Variável & Grupo & Média & DP & Mínimo & Máximo & $1 Q$ & Mediana & $3 Q$ & $\begin{array}{l}\text { valor } \\
\text { de } p\end{array}$ \\
\hline \multirow[t]{2}{*}{ ESV Limitação } & G1 & 8,30 & 7,33 & 0,00 & 26,00 & 2,00 & 8,00 & 10,25 & 0,218 \\
\hline & G2 & 10,87 & 9,15 & 0,00 & 37,00 & 3,50 & 10,00 & 15,25 & \\
\hline \multirow[t]{2}{*}{ ESV Físico } & G1 & 6,33 & 4,52 & 0,00 & 20,00 & 2,75 & 5,50 & 8,50 & 0,649 \\
\hline & G2 & 5,73 & 4,13 & 0,00 & 17,00 & 2,00 & 5,00 & 8,25 & \\
\hline \multirow[t]{2}{*}{ ESV Emocional } & G1 & 1,13 & 2,96 & 0,00 & 14,00 & 0,00 & 0,00 & 0,50 & 0,185 \\
\hline & G2 & 2,70 & 4,85 & 0,00 & 18,00 & 0,00 & 0,00 & 4,00 & \\
\hline \multirow[t]{2}{*}{ ESV Total } & G1 & 15,77 & 12,51 & 0,00 & 54,00 & 7,00 & 14,00 & 20,00 & 0,391 \\
\hline & G2 & 19,30 & 16,05 & 2,00 & 63,00 & 7,50 & 16,00 & 25,00 & \\
\hline \multirow[t]{2}{*}{ Frequência queimação } & G1 & 0,43 & 1,04 & 0,00 & 4,00 & 0,00 & 0,00 & 0,00 & 0,453 \\
\hline & G2 & 0,47 & 0,90 & 0,00 & 4,00 & 0,00 & 0,00 & 1,00 & \\
\hline \multirow[t]{2}{*}{ Frequência aperto } & G1 & 0,30 & 0,70 & 0,00 & 3,00 & 0,00 & 0,00 & 0,00 & 0,734 \\
\hline & G2 & 0,37 & 0,76 & 0,00 & 3,00 & 0,00 & 0,00 & 0,25 & \\
\hline \multirow[t]{2}{*}{ Frequência secura } & G1 & 0,93 & 0,94 & 0,00 & 4,00 & 0,00 & 1,00 & 1,00 & 0,550 \\
\hline & G2 & 0,77 & 0,77 & 0,00 & 3,00 & 0,00 & 1,00 & 1,00 & \\
\hline \multirow[t]{2}{*}{ Frequência garganta dolorida } & G1 & 0,53 & 0,63 & 0,00 & 2,00 & 0,00 & 0,00 & 1,00 & 0,403 \\
\hline & G2 & 0,43 & 0,68 & 0,00 & 2,00 & 0,00 & 0,00 & 1,00 & \\
\hline \multirow[t]{2}{*}{ Frequência coceira } & G1 & 0,60 & 1,00 & 0,00 & 4,00 & 0,00 & 0,00 & 1,00 & 0,223 \\
\hline & G2 & 0,80 & 1,06 & 0,00 & 5,00 & 0,00 & 1,00 & 1,00 & \\
\hline \multirow[t]{2}{*}{ Frequência garganta sensível } & G1 & 0,30 & 0,47 & 0,00 & 1,00 & 0,00 & 0,00 & 1,00 & 0,483 \\
\hline & G2 & 0,43 & 0,63 & 0,00 & 2,00 & 0,00 & 0,00 & 1,00 & \\
\hline \multirow[t]{2}{*}{ Frequência garganta irritada } & G1 & 0,50 & 0,78 & 0,00 & 3,00 & 0,00 & 0,00 & 1,00 & 0,474 \\
\hline & G2 & 0,53 & 0,57 & 0,00 & 2,00 & 0,00 & 0,50 & 1,00 & \\
\hline \multirow[t]{2}{*}{ Frequência bola na garganta } & G1 & 0,57 & 1,19 & 0,00 & 4,00 & 0,00 & 0,00 & 0,25 & 0,757 \\
\hline & G2 & 0,27 & 0,52 & 0,00 & 2,00 & 0,00 & 0,00 & 0,25 & \\
\hline \multirow[t]{2}{*}{ EDTV frequência } & G1 & 4,17 & 4,68 & 0,00 & 18,00 & 1,00 & 3,00 & 5,25 & 0,526 \\
\hline & G2 & 4,07 & 3,54 & 0,00 & 15,00 & 2,00 & 4,00 & 5,25 & \\
\hline \multirow[t]{2}{*}{ Intensidade queimação } & G1 & 0,43 & 1,14 & 0,00 & 4,00 & 0,00 & 0,00 & 0,00 & 0,423 \\
\hline & G2 & 0,47 & 1,04 & 0,00 & 5,00 & 0,00 & 0,00 & 1,00 & \\
\hline \multirow[t]{2}{*}{ Intensidade aperto } & G1 & 0,23 & 0,50 & 0,00 & 2,00 & 0,00 & 0,00 & 0,00 & 0,718 \\
\hline & G2 & 0,30 & 0,60 & 0,00 & 2,00 & 0,00 & 0,00 & 0,25 & \\
\hline \multirow[t]{2}{*}{ Intensidade secura } & G1 & 0,80 & 0,76 & 0,00 & 3,00 & 0,00 & 1,00 & 1,00 & 0,674 \\
\hline & G2 & 0,73 & 0,78 & 0,00 & 3,00 & 0,00 & 1,00 & 1,00 & \\
\hline \multirow[t]{2}{*}{ Intensidade garganta dolorida } & G1 & 0,43 & 0,68 & 0,00 & 2,00 & 0,00 & 0,00 & 1,00 & 0,575 \\
\hline & G2 & 0,37 & 0,72 & 0,00 & 3,00 & 0,00 & 0,00 & 1,00 & \\
\hline \multirow[t]{2}{*}{ Intensidade coceira } & G1 & 0,53 & 1,01 & 0,00 & 4,00 & 0,00 & 0,00 & 1,00 & 0,094 \\
\hline & G2 & 0,77 & 0,94 & 0,00 & 4,00 & 0,00 & 1,00 & 1,00 & \\
\hline \multirow[t]{2}{*}{ Intensidade garganta sensível } & G1 & 0,33 & 0,55 & 0,00 & 2,00 & 0,00 & 0,00 & 1,00 & 1,000 \\
\hline & G2 & 0,33 & 0,55 & 0,00 & 2,00 & 0,00 & 0,00 & 1,00 & \\
\hline \multirow[t]{2}{*}{ Intensidade garganta irritada } & G1 & 0,60 & 1,00 & 0,00 & 4,00 & 0,00 & 0,00 & 1,00 & 0,781 \\
\hline & G2 & 0,43 & 0,68 & 0,00 & 3,00 & 0,00 & 0,00 & 1,00 & \\
\hline \multirow[t]{2}{*}{ Intensidade bola na garganta } & G1 & 0,40 & 0,97 & 0,00 & 4,00 & 0,00 & 0,00 & 0,00 & 0,660 \\
\hline & G2 & 0,33 & 0,61 & 0,00 & 2,00 & 0,00 & 0,00 & 1,00 & \\
\hline \multirow[t]{2}{*}{ EDTV intensidade } & G1 & 3,77 & 4,30 & 0,00 & 17,00 & 0,00 & 3,00 & 5,25 & 0,551 \\
\hline & G2 & 3,73 & 3,37 & 0,00 & 13,00 & 1,00 & 3,00 & 5,25 & \\
\hline
\end{tabular}

Teste de Mann-Whitney

Legenda: G1 = Grupo 1; G2 = Grupo 2; DP = desvio padrão; $1 \mathrm{Q}=$ primeiro quartil; $3 \mathrm{Q}$ = terceiro quartil; ESV = Escala de Sintomas Vocais; EDTV = Escala de Desconforto no Trato Vocal

que administravam insulinoterapia por meio de aplicações ao longo do dia (90\%). Neste grupo, observou-se correlação positiva moderada entre o tempo de diagnóstico da DM1 e a frequência de queimação $(r=0,575 ; p=0,001)$, frequência de garganta sensível $(r=0,518 ; \mathrm{p}=0,003) \mathrm{e}$ intensidade de queimação $(\mathrm{r}=0,516 ; \mathrm{p}=0,004)($ Tabela 2$)$.
Também foram observados, no G1, valores significativamente maiores de frequência de garganta sensível $(\mathrm{p}=0,006)$, frequência de garganta irritada $(\mathrm{p}=0,004)$, intensidade de garganta sensível $(p=0,003)$ e intensidade de garganta irritada $(p=0,004)$, em indivíduos que relataram realizar insulinoterapia por meio do uso de bomba de infusão (Tabela 3). 
Tabela 2. Correlação entre tempo de diagnóstico e a autoavaliação de sintomas vocais, do desconforto no trato vocal e da autoavaliação vocal em indivíduos com diabete mellitus tipo 1 (Grupo 1)

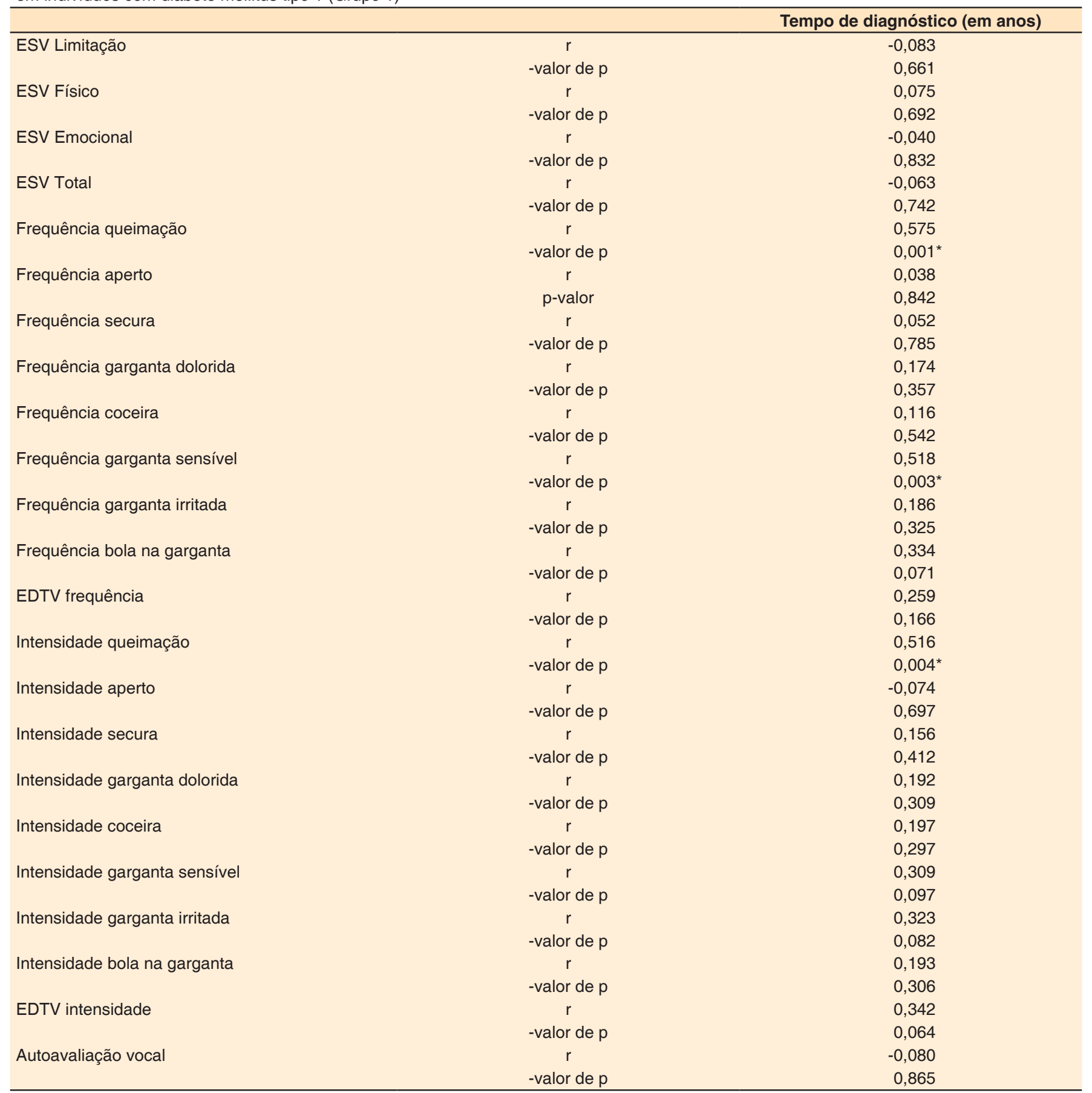

Teste de Correlação de Spearman; ${ }^{*} \mathrm{p}<0,05$;

Legenda: $r$ = coeficiente de correlação; ESV = Escala de Sintomas Vocais; EDTV = Escala de Desconforto no Trato Vocal

Tabela 3. Comparação da autoavaliação de sintomas vocais e do desconforto no trato vocal em função da forma de administração de insulinoterapia em indivíduos com diabetes mellitus tipo 1 (Grupo 1)

\begin{tabular}{llcccccccc}
\hline \multicolumn{1}{c}{ Variável } & \multicolumn{1}{c}{$\begin{array}{c}\text { Tipo de } \\
\text { Insulinoterapia }\end{array}$} & Média & DP & Mínimo & Máximo & $\mathbf{1 Q}$ & Mediana & $\mathbf{3 Q}$ & $\begin{array}{c}\text { valor } \\
\text { de } \mathbf{p}\end{array}$ \\
\hline ESV Limitação & Bomba de Infusão & 7,33 & 4,62 & 2,00 & 10,00 & 2,00 & 10,00 & 0,00 & 0,754 \\
& Aplicação & 8,41 & 7,63 & 0,00 & 26,00 & 2,00 & 8,00 & 11,00 & \\
ESV Físico & Bomba de Infusão & 7,67 & 2,52 & 5,00 & 10,00 & 5,00 & 8,00 & 0,00 & 0,330 \\
& Aplicação & 6,19 & 4,70 & 0,00 & 20,00 & 2,00 & 5,00 & 8,00 & \\
\hline
\end{tabular}

Teste de Mann-Whitney; ${ }^{*} \mathrm{p}<0,05$;

Legenda: $\mathrm{DP}$ = desvio padrão; $1 \mathrm{Q}=$ primeiro quartil; $3 \mathrm{Q}=$ terceiro quartil; ESV = Escala de Sintomas Vocais; EDTV = Escala de Desconforto no Trato Vocal 
Tabela 3. Continuação...

\begin{tabular}{|c|c|c|c|c|c|c|c|c|c|}
\hline Variável & $\begin{array}{c}\text { Tipo de } \\
\text { Insulinoterapia }\end{array}$ & Média & DP & Mínimo & Máximo & $1 Q$ & Mediana & $3 Q$ & $\begin{array}{l}\text { valor } \\
\text { de } p\end{array}$ \\
\hline \multirow[t]{2}{*}{ ESV Emocional } & Bomba de Infusão & 0,00 & 0,00 & 0,00 & 0,00 & 0,00 & 0,00 & 0,00 & 0,327 \\
\hline & Aplicação & 1,26 & 3,10 & 0,00 & 14,00 & 0,00 & 0,00 & 2,00 & \\
\hline \multirow[t]{2}{*}{ ESV Total } & Bomba de Infusão & 15,00 & 5,00 & 10,00 & 20,00 & 10,00 & 15,00 & 0,00 & 0,628 \\
\hline & Aplicação & 15,85 & 13,14 & 0,00 & 54,00 & 7,00 & 14,00 & 20,00 & \\
\hline \multirow{2}{*}{$\begin{array}{l}\text { Frequência } \\
\text { queimação }\end{array}$} & Bomba de Infusão & 0,33 & 0,58 & 0,00 & 1,00 & 0,00 & 0,00 & 0,00 & 0,656 \\
\hline & Aplicação & 0,44 & 1,09 & 0,00 & 4,00 & 0,00 & 0,00 & 0,00 & \\
\hline \multirow[t]{2}{*}{ Frequência aperto } & Bomba de Infusão & 0,00 & 0,00 & 0,00 & 0,00 & 0,00 & 0,00 & 0,00 & 0,372 \\
\hline & Aplicação & 0,33 & 0,73 & 0,00 & 3,00 & 0,00 & 0,00 & 0,00 & \\
\hline \multirow[t]{2}{*}{ Frequência secura } & Bomba de Infusão & 1,67 & 2,08 & 0,00 & 4,00 & 0,00 & 1,00 & 0,00 & 0,597 \\
\hline & Aplicação & 0,85 & 0,77 & 0,00 & 3,00 & 0,00 & 1,00 & 1,00 & \\
\hline \multirow{2}{*}{$\begin{array}{l}\text { Frequência garganta } \\
\text { dolorida }\end{array}$} & Bomba de Infusão & 0,67 & 0,58 & 0,00 & 1,00 & 0,00 & 1,00 & 0,00 & 0,585 \\
\hline & Aplicação & 0,52 & 0,64 & 0,00 & 2,00 & 0,00 & 0,00 & 1,00 & \\
\hline \multirow[t]{2}{*}{ Frequência coceira } & Bomba de Infusão & 1,00 & 1,73 & 0,00 & 3,00 & 0,00 & 0,00 & 0,00 & 0,840 \\
\hline & Aplicação & 0,56 & 0,93 & 0,00 & 4,00 & 0,00 & 0,00 & 1,00 & \\
\hline \multirow{2}{*}{$\begin{array}{l}\text { Frequência garganta } \\
\text { sensível }\end{array}$} & Bomba de Infusão & 1,00 & 0,00 & 1,00 & 1,00 & 1,00 & 1,00 & 1,00 & $0,006^{*}$ \\
\hline & Aplicação & 0,22 & 0,42 & 0,00 & 1,00 & 0,00 & 0,00 & 0,00 & \\
\hline \multirow{2}{*}{$\begin{array}{l}\text { Frequência garganta } \\
\text { irritada }\end{array}$} & Bomba de Infusão & 2,00 & 1,00 & 1,00 & 3,00 & 1,00 & 2,00 & 0,00 & $0,004^{*}$ \\
\hline & Aplicação & 0,33 & 0,55 & 0,00 & 2,00 & 0,00 & 0,00 & 1,00 & \\
\hline \multirow{2}{*}{$\begin{array}{l}\text { Frequência bola na } \\
\text { garganta }\end{array}$} & Bomba de Infusão & 0,00 & 0,00 & 0,00 & 0,00 & 0,00 & 0,00 & 0,00 & 0,327 \\
\hline & Aplicação & 0,63 & 1,24 & 0,00 & 4,00 & 0,00 & 0,00 & 1,00 & \\
\hline \multirow[t]{2}{*}{ EDTV frequência } & Bomba de Infusão & 6,67 & 3,21 & 3,00 & 9,00 & 3,00 & 8,00 & 0,00 & 0,153 \\
\hline & Aplicação & 3,89 & 4,77 & 0,00 & 18,00 & 1,00 & 3,00 & 4,00 & \\
\hline \multirow{2}{*}{$\begin{array}{l}\text { Intensidade } \\
\text { queimação }\end{array}$} & Bomba de Infusão & 0,33 & 0,58 & 0,00 & 1,00 & 0,00 & 0,00 & 0,00 & 0,523 \\
\hline & Aplicação & 0,44 & 1,19 & 0,00 & 4,00 & 0,00 & 0,00 & 0,00 & \\
\hline \multirow[t]{2}{*}{ Intensidade aperto } & Bomba de Infusão & 0,00 & 0,00 & 0,00 & 0,00 & 0,00 & 0,00 & 0,00 & 0,371 \\
\hline & Aplicação & 0,26 & 0,53 & 0,00 & 2,00 & 0,00 & 0,00 & 0,00 & \\
\hline \multirow[t]{2}{*}{ Intensidade secura } & Bomba de Infusão & 1,33 & 1,53 & 0,00 & 3,00 & 0,00 & 1,00 & 0,00 & 0,518 \\
\hline & Aplicação & 0,74 & 0,66 & 0,00 & 2,00 & 0,00 & 1,00 & 1,00 & \\
\hline \multirow{2}{*}{$\begin{array}{l}\text { Intensidade garganta } \\
\text { dolorida }\end{array}$} & Bomba de Infusão & 1,00 & 1,00 & 0,00 & 2,00 & 0,00 & 1,00 & 0,00 & 0,157 \\
\hline & Aplicação & 0,37 & 0,63 & 0,00 & 2,00 & 0,00 & 0,00 & 1,00 & \\
\hline \multirow[t]{2}{*}{ Intensidade coceira } & Bomba de Infusão & 1,33 & 2,31 & 0,00 & 4,00 & 0,00 & 0,00 & 0,00 & 0,638 \\
\hline & Aplicação & 0,44 & 0,80 & 0,00 & 3,00 & 0,00 & 0,00 & 1,00 & \\
\hline \multirow{2}{*}{$\begin{array}{l}\text { Intensidade garganta } \\
\text { sensível }\end{array}$} & Bomba de Infusão & 1,33 & 0,58 & 1,00 & 2,00 & 1,00 & 1,00 & 0,00 & $0,003^{*}$ \\
\hline & Aplicação & 0,22 & 0,42 & 0,00 & 1,00 & 0,00 & 0,00 & 0,00 & \\
\hline \multirow{2}{*}{$\begin{array}{l}\text { Intensidade garganta } \\
\text { irritada }\end{array}$} & Bomba de Infusão & 2,67 & 1,53 & 1,00 & 4,00 & 1,00 & 3,00 & 0,00 & $0,004^{*}$ \\
\hline & Aplicação & 0,37 & 0,63 & 0,00 & 2,00 & 0,00 & 0,00 & 1,00 & \\
\hline \multirow{2}{*}{$\begin{array}{l}\text { Intensidade bola na } \\
\text { garganta }\end{array}$} & Bomba de Infusão & 0,00 & 0,00 & 0,00 & 0,00 & 0,00 & 0,00 & 0,00 & 0,373 \\
\hline & Aplicação & 0,44 & 1,01 & 0,00 & 4,00 & 0,00 & 0,00 & 0,00 & \\
\hline \multirow[t]{2}{*}{ EDTV intensidade } & Bomba de Infusão & 8,00 & 4,36 & 3,00 & 11,00 & 3,00 & 10,00 & 0,00 & 0,086 \\
\hline & Aplicação & 3,30 & 4,11 & 0,00 & 17,00 & 0,00 & 2,00 & 5,00 & \\
\hline
\end{tabular}

Teste de Mann-Whitney; * $\mathrm{p}<0,05$;

Legenda: $\mathrm{DP}=$ desvio padrão; $1 \mathrm{Q}=$ primeiro quartil; $3 \mathrm{Q}=$ terceiro quartil; ESV = Escala de Sintomas Vocais; EDTV = Escala de Desconforto no Trato Vocal

\section{DISCUSSÃO}

Na literatura, a DM1 é associada com patologias, como refluxo laringofaríngeo $(\mathrm{RLF})^{(6)}$ e distúrbio sensorial laríngeo ${ }^{(6,7)}$. Além disso, há relato de associação da DM com sintomas ou desconfortos no trato vocal ${ }^{(6)}$. Desta forma, vê-se a necessidade de investigar melhor a autoavaliação de sintomas e desconforto no trato vocal em indivíduos com e sem DM1, bem como a influência de característica da DM1 na autoavaliação.

Não houve diferença entre os grupos para a sintomatologia vocal. A autoavaliação de sintomas vocais mostrou valores de tendência central medianos iguais ou inferiores ao ponto de corte da ESV, para todos os domínios, em ambos os grupos ${ }^{(12)}$. Sendo assim, em nenhum dos grupos a tendência central esteve dentro da faixa de risco para o desenvolvimento de distúrbios vocais.

Também não foi encontrada diferença entre os grupos para a autoavaliação de desconforto no trato vocal. A EDTV é apenas traduzida para o português brasileiro ${ }^{(10)} \mathrm{e}$ não possui ponto de corte para diferenciar indivíduos com e sem riscos para desenvolver distúrbios vocais, o que impossibilita a análise dos valores de tendência central. No entanto, uma pesquisa que aplicou a EDTV em um grupo controle sem queixas de voz obteve valores de tendência central bastante semelhantes aos resultados obtidos neste estudo, tanto para a frequência, quanto para a intensidade ${ }^{(13)}$. Deste modo, pode-se inferir que os resultados das respostas dos diabéticos para esse 
instrumento são compatíveis aos apresentados por pessoas vocalmente saudáveis.

De modo geral, os dados encontrados para autopercepção de sintomas e de desconforto no trato vocal apontaram que a autopercepção foi baixa em ambos os grupos e que não resultaram em uma influência perceptível no dia a dia dos participantes. Desta forma, constata-se que apenas o fato de possuir DM1 não parece, necessariamente, configurar risco para desenvolver sintomas ou desconforto no trato vocal. Tais dados parecem estar mais relacionados com as características da DM1.

Observou-se correlação positiva moderada entre o tempo de diagnóstico da DM1 e a frequência e a intensidade de queimação e a frequência de garganta sensível. É importante ressaltar que a DM1 é uma doença progressiva, o que pode estabelecer relação com o tempo de diagnóstico. Outros estudos apontaram relação entre a duração de sintomas gastrointestinais e o tempo de diagnóstico da DM. Autores relacionaram sintomas de garganta sensível e queimação ao RLF, que é uma patologia frequente em indivíduos com $\mathrm{DM}^{(6)}$. Neste caso, os sintomas de aumento da queimação e sensibilidade seria decorrentes de um fraco mecanismo de defesa da mucosa laríngea e faríngea contra o ácido e a pepsina refluídos no $\mathrm{RLF}^{(6)}$.

Observaram-se valores significativamente maiores de frequência e intensidade de garganta sensível e de garganta irritada em indivíduos que relataram realizar insulinoterapia por meio do uso de bomba de infusão, em comparação aos que referiram fazer aplicações ao longo do dia. É importante ressaltar que há chance de erro tipo I no presente resultado, podendo existir um fator aleatório que tenha interferido no resultado, visto que o tamanho de amostra do grupo que utilizou bomba de infusão foi pequeno.

Uma revisão sistemática que comparou as duas modalidades de insulinoterapia apontou que a terapia com bomba de insulina é desnecessária para a maioria das pessoas com DM1 e deve ser reservada para pessoas com problemas especiais com injeções de insulina $^{(14)}$. Outro estudo acrescentou, ainda, que a insulinoterapia por meio de bomba de infusão é uma modalidade de terapia que tem se mostrado particularmente útil naqueles pacientes com DM de difícil controle, que apresentam episódios repetidos de cetoacidose diabética ou hipoglicemias ${ }^{(6)}$.

Um estudo relatou a possível disfunção sensorial da laringe, que gera uma hipersensibilidade neuronal na laringe ${ }^{(6)}$, decorrente da hipoglicemia prolongada, que causa alterações degenerativas em grandes axônios mielinizados do nervo laríngeo recorrente ${ }^{(7)}$. Portanto, ainda há pouca clareza sobre a explicação desse achado, porém, infere-se que possa haver uma relação entre a maior ocorrência de disfunção sensorial da laringe de indivíduos que realizam a terapia com bomba de infusão subcutânea, decorrente da maior ocorrência de episódios de hipoglicemia nesses pacientes ${ }^{(7)}$.

Apesar de não ter existido diferença na percepção de sintomas vocais e do desconforto do trato vocal entre indivíduos com e sem DM1, a forma de administração de insulinoterapia e o tempo de diagnóstico influenciaram na percepção de desconfortos no trato vocal. $\mathrm{O}$ presente estudo apresentou limitações quanto à amostra por conveniência. Assim, são necessários novos estudos para analisar a associação entre as características da DM1, os sintomas gastrointestinais, a disfunção laríngea e as medidas clínicas e subjetivas de voz e laringe, nessa população.

\section{CONCLUSÃO}

Indivíduos com DM1 apresentam baixa sintomatologia vocal e desconforto no trato vocal, além de não se diferenciarem do grupo controle. Porém, características da doença, como tempo de diagnóstico e forma de administração de insulinoterapia influenciam sua percepção da frequência e da intensidade de desconforto no trato vocal.

\section{REFERÊNCIAS}

1. Santos JR, Enumo SRF. Adolescentes com Diabetes mellitus tipo 1: seu cotidiano e enfrentamento da doença. Psicol Reflex Crit. 2003;16(2):41125. http://dx.doi.org/10.1590/S0102-79722003000200021.

2. Gross JL, Nehme M. Detecção e tratamento das complicações crônicas do diabetes melito: Consenso da Sociedade Brasileira de Diabetes e Conselho Brasileiro de Oftalmologia. Rev Assoc Med Bras. 1999;45(3):279-84. http://dx.doi.org/10.1590/S0104-42301999000300014. PMid:10513061.

3. Lee JJ, Thompson MJ, Usher-Smith JA, Koshiaris C, Van den Bruel A. Opportunities for earlier diagnosis of type 1 diabetes in children: a case-control study using routinely collected primary care records. Prim Care Diabetes. 2018;12(3):254-64. http://dx.doi.org/10.1016/j. pcd.2018.02.002. PMid:29548694.

4. Sales-Peres SHC, Guedes MFS, Sá LM, Negrato CA, Lauris JRP. Estilo de vida em pacientes portadores de diabetes mellitus tipo 1: uma revisão sistemática. Cien Saude Colet. 2016;21(4):1197-206. http:// dx.doi.org/10.1590/1413-81232015214.20242015. PMid:27076018.

5. Kirchner LF, Marinho-Casanova ML. Avaliação da adesão ao tratamento do diabetes mellitus tipo 1: revisão de literatura. Estud Interdiscip Psicol. 2014;5(1):45. http://dx.doi.org/10.5433/2236-6407.2014v5n1p45.

6. Hamdan A, Jabbour J, Barazi R, Korban Z, Azar ST. Prevalence of laryngopharyngeal reflux disease in patients with diabetes mellitus. $\mathrm{J}$ Voice. 2013;27(4):495-9. http://dx.doi.org/10.1016/j.jvoice.2012.07.010. PMid:23602088.

7. Jamali R, Mohseni S. Hypoglycaemia causes degeneration of large myelinated nerve fibres in the vagus nerve of insulin-treated diabetic BB/Wor rats. Acta Neuropathol. 2005;109(2):198-206. http://dx.doi. org/10.1007/s00401-004-0932-1. PMid:15549329.

8. Moreti F, Zambon F, Oliveira G, Behlau M. Cross-cultural adaptation, validation, and cutoff values of the Brazilian version of the Voice Symptom Scale: VoiSS. J Voice. 2014;28(4):458-68. http://dx.doi. org/10.1016/j.jvoice.2013.11.009. PMid:24560004.

9. Mathieson L. Vocal tract discomfort in hyperfunctional dysphonia. J Voice. 1993;2:40-8.

10. Rodrigues G, Zambon F, Mathieson L, Behlau M. Vocal tract discomfort in teachers: its relationship to self-reported voice disorders. J Voice. 2013;27(4):473-80. http://dx.doi.org/10.1016/j.jvoice.2013.01.005. PMid:23528674.

11. Landis J, Koch G. The measurement of observer agreement for categorical data. Biometrics. 1977;33(1):159-74. http://dx.doi. org/10.2307/2529310. PMid:843571.

12. Behlau M, Madazio G, Moreti F, Oliveira G, Santos LM, Paulinelli BR, et al. Efficiency and Cutoff Values of Self-Assessment Instruments on the Impact of a Voice Problem. J Voice. 2016;30(4):506.e9-18. http://dx.doi.org/10.1016/j.jvoice.2015.05.022. PMid:26168902.

13. Amaral AC, Zambon F, Moreti F, Behlau M. Desconforto do trato vocal em professores após atividade letiva. CoDAS. 2017;29(2):1-7. http://dx.doi.org/10.1590/2317-1782/20172016045.

14. Pickup J, Mattock M, Kerry S. Glycaemic control with continuous subcutaneous insulin infusion compared with intensive insulin injections in patients with type 1 diabetes: meta-analysis of randomised controlled trials. BMJ. 2002;324(7339):705-705. http://dx.doi.org/10.1136/ bmj.324.7339.705. PMid:11909787. 